\title{
Gaeumannomyces graminis vars. avenae, graminis, and tritici Identified Using PCR Amplification of Avenacinase-like Genes
}

\author{
Sansanalak Rachdawong, School of Bioresources and Technology, King Mongkut's University of Technology \\ Thonburi, Bangkok 10140, Thailand; and Carole L. Cramer, Elizabeth A. Grabau, Verlyn K. Stromberg, \\ George H. Lacy, and Erik L. Stromberg, Department of Plant Pathology, Physiology, and Weed Science, Virginia \\ Polytechnic Institute and State University, Blacksburg 24061-0330
}

\begin{abstract}
Rachdawong, S., Cramer, C. L., Grabau, E. A., Stromberg, V. K., Lacy, G. H., and Stromberg, E. L. 2002. Gaeumannomyces graminis vars. avenae, graminis, and tritici identified using PCR amplification of avenacinase-like genes. Plant Dis. 86:652-660.

Identifying take-all pathogens, Gaeumannomyces graminis varieties avenae (Gga), graminis $(G g g)$, and tritici $(G g t)$, is difficult. Rapid identification is important for development of disease thresholds. We developed a single-tube, polymerase chain reaction (PCR) method differentiating among $G g a, G g g$, and Ggt. Nucleotide base sequence analyses of avenacinase-like genes from $G g a, G g g$, and $G g t$ isolates provided the basis for designing variety-specific primers. Sequences from $G g g$ and $G g t$ were highly related (99\% identity), but $G g a$ sequences were $<95 \%$ identical to Ggg and Ggt sequences. Three $5^{\prime}$ primers specific for Gga, Ggt, and Ggg and a single $3^{\prime}$ common primer allowed amplification of variety-specific fragments of 617,870 , and $1,086 \mathrm{bp}$, respectively. Each $5^{\prime}$ primer was specific in mixed populations of primers and templates. No PCR products were amplified from related fungi including Gaeumannomyces cylindrosporus and Phialophora spp. We surveyed 16 putative Ggt isolates using our assay; nine produced $G g t$-specific fragments and seven produced $G g g$-specific fragments. Five Gga isolates produced $G g a$-specific fragments. However, Gga- and $G g t$-specific fragments were observed from a sixth $G g a$ isolate, RB-W, which indicates a mixed culture or a heterokaryon. Our singletube, PCR method rapidly differentiates among the important take-all pathogens commonly encountered together in cereal fields.
\end{abstract}

Additional keywords: avenacin, Avenae sativa, grasses, oats, phylogenetic relatedness, Triticum aestivum, wheat

Gaeumannomyces graminis (Sacc.) Arx \& Olivier, a soilborne ascomycete, is the causal agent of take-all of cereal and grasses worldwide. The fungus colonizes the roots of susceptible plants, producing symptoms including stunted shoot growth, white-heads, reduced and blackened roots, and premature grain ripening. Based on pathogenicity assays and cultural characteristics, three varieties of $G$. graminis have been recognized (33). G. graminis var. tritici Walker $(G g t)$ causes take-all of wheat (Triticum aestivum L.), barley (Hordeum vulgare $\mathrm{L}$.$) , rye (Secale cereale L.),$ and, occasionally, oats (Avenae sativa L.) $(4,38)$, and is the most economically important take-all pathogen $(6,20)$. Other varieties of $G$. graminis may also colonize wheat roots and commonly occur in wheat fields on weeds. G. graminis var. avenae (E.M. Turner) Dennis $(G g a)$ is a pathogen of oats and causes take-all patch of

Corresponding author: George H. Lacy

E-mail: lacygh@vt.edu

Accepted for publication 5 February 2002.

Publication no. D-2002-0412-02R

(C) 2002 The American Phytopathological Society turfgrasses (34), and may affect wheat (33). G. graminis var. graminis ( $G g g$ ) is pathogenic on cereals and grasses $(12,33)$, causes crown sheath rot of rice (Oryza sativa $\mathrm{L}$.), and is weakly virulent on wheat (34). To complicate the situation, other species of Gaeumannomyces and morphologically similar members of the genus Phialophora (Gaeumannomyces-Phialophora complex) are capable of colonizing and causing root rot on cereals and grasses. For example, G. cylindrosporus and $G$. incrustans cause root rots when inoculated on wheat, barley, and turfgrasses but are not considered to be pathogens in field situations $(21,24)$.

For predicting disease loss thresholds, epidemiology studies, and estimating the effectiveness of disease control strategies, a need exists for reliable identification of the three varieties of $G$. graminis among the Gaeumannomyces-Phialophora complex and other soil and rhizosphere organisms. Conventional identification methods for distinguishing $G$. graminis varieties are laborious, time-consuming, and often inconclusive. Identification is based on a combination of the characteristic disease symptoms, perithecia and ascospore morphological characters and size, hyphopodia type, and pathogenicity assays (20). While
Ggt and Gga produce simple and nonlobed hyphopodia, Ggg and Phialophora spp. produce hyphopodia that are lobed or nonlobed $(9,13,26)$. Variation in size of the ascospores can also be used to differentiate among the three varieties; however, overlapping size ranges makes specific identification difficult or inconclusive. Pathogenicity assays are time-consuming and sometimes difficult to interpret because the organism in question may lose virulence during long-term storage (1) or may only colonize or even be weakly virulent on "nonhost" or "host" plants (12,21,24,33,34).

Selective media have made isolation of $G$. graminis more effective, but they are not useful for identifying the most important varieties, Gga, Ggg, and Ggt (23). Further, during isolation, these slowgrowing fungi are easily overgrown by other organisms.

Molecular techniques have played a significant role in the recent development of specific and rapid identification tests for $G$. graminis, especially Ggt. Previous work focused on the use of restriction fragment length polymorphisms (RFLPs) $(2,17,32,36)$, amplification of mitochondrial DNA fragments $(11,18,31)$, amplification of specific sequences within the internal transcribed spacer (ITS) region of ribosomal DNA (rDNA; 4,16,27), or randomly amplified polymorphic DNA (RAPD) profiles $(5,15,37)$. Recently, polymerase chain reaction (PCR) primers have been developed for a fragment of the 18S rDNA gene and allow amplification of distinct $G g a$ and Ggt products (14). However, no Ggg products were amplified using these primers.

To develop a rapid and definitive identification method for differentiation among G. graminis varieties $G g a, G g g$, and Ggt, we proposed to utilize nucleotide base differences in avenacinase-like genes to design variety-specific PCR primers. Avenacinase-like genes are of special interest because at least one gene is involved in a specific host-pathogen interaction (7). We reasoned that genes important to the selectivity of plant-fungus interactions would require conservation of specific sequences. Avenacinase produced by $G g a$ detoxifies avenacin A-1, the most abundant fungitoxic substance present in oat roots (7). The ability to detoxify avenacin A-1 is a pathogenicity factor for $G g a$ on oats 
(7,29). Ggt and $G g g$ also have the ability to produce avenacinase-like proteins, but they do not affect avenacin A-1 (7,29,30), contributing to the inability of these fungi to cause disease on oats. Our objectives were to isolate and sequence avenacinase-like genes from $G g a, G g g$, and $G g t$, to determine if there was adequate divergence at the nucleic acid level to allow variety differentiation, and using simple PCR protocols, to amplify variety-specific bands.

\section{MATERIALS AND METHODS}

Fungal isolates, maintenance, and cultivation. All fungi used in this study are listed in Table 1. Gaeumannomyces spp. and Phialospora spp. isolates were cultured at $25^{\circ} \mathrm{C}$ and stored at $4{ }^{\circ} \mathrm{C}$ on dilute $(0.25 \times)$ potato dextrose agar (PDA; Sigma, St. Louis, MO). For long-term storage, mycelium plugs were taken from the colony margin using a cork borer (no. 3; 8 $\mathrm{mm}$ diameter) and stored at $-80^{\circ} \mathrm{C}$ in $15 \%$ glycerol. Isolates other than those from the American Type Culture Collection (ATCC, Manassas, VA) were purified by single hyphal tip isolation prior to DNA work.

For fungal cultivation for DNA isolation, three mycelial plugs were taken from the colony margin using a cork borer (no. 2; $5 \mathrm{~mm}$ diameter) and inoculated into 50 $\mathrm{ml}$ of $0.25 \times$ potato dextrose broth (PDB; Sigma) in a $250-\mathrm{ml}$ flask and grown in an orbital incubator for 7 days at $25^{\circ} \mathrm{C}, 120$ $\mathrm{rpm}$. Resulting mycelia were chopped in a blender and transferred to a 1-liter flask containing 250 to $300 \mathrm{ml}$ of $0.25 \times$ PDB, and incubation was continued as previously described for 7 to 10 days.

Preparation of fungal DNA. Mycelia were harvested from PDB on four layers of cheesecloth and transferred onto sterile Whatman No. 1 filter paper. Wet mycelia were lyophilized overnight, collected, and stored in a desiccator at room temperature until used. Fungal DNA was prepared according to the protocol of Lee and Taylor (25). Approximately $1 \mathrm{~g}$ of dry mycelia was ground in liquid nitrogen, immediately mixed with $10 \mathrm{ml}$ of lysis buffer $(50 \mathrm{mM}$ Tris-HCl, pH 7.2; 50 mM EDTA; 3\% wt/vol sodium dodecyl sulfate (SDS); and $1 \% \mathrm{vol} / \mathrm{vol} \beta$-mercaptoethanol), stirred with a 5-ml pipette tip, vortexed vigorously until homogeneous, incubated for 1 to $2 \mathrm{~h}$ at $65^{\circ} \mathrm{C}$, extracted with phenol:chloroform $(1: 1 \mathrm{vol} / \mathrm{vol})$ until the aqueous phase was clear, incubated with RNase A (final concentration of $50 \mu \mathrm{g} / \mathrm{ml}$ ) at $37^{\circ} \mathrm{C}$ for $3 \mathrm{~h}$, reextracted once with phenol:chloroform, and precipitated with 0.03 vol of $3 \mathrm{M}$ sodium acetate and $0.6 \mathrm{vol}$ of $99 \%$ isopropanol at $4^{\circ} \mathrm{C}$ for at least $30 \mathrm{~min}$. The DNA was pelleted by centrifugation for $20 \mathrm{~min}$ $\left(14,000 \times g, 25^{\circ} \mathrm{C}\right)$, rinsed with $70 \%$ ethanol, dried, and redissolved in Tris-EDTA (TE) or deionized water. DNA solutions were stored at $4{ }^{\circ} \mathrm{C}$.

Cloning avenacinase-like genes. About $1.4 \mathrm{~kb}$ of the $5^{\prime}$ ends of avenacinase-like genes from Ggt and Ggg isolates were amplified by PCR using oligonucleotide primers AV1 and AV3 (see Figure 1 for annealing sites and primer sequences) synthesized by Integrated DNA Technologies, Inc. (Coralville, IA). PCR reactions were performed in $50-\mu$ l reaction volumes containing $50 \mathrm{pmol}$ of each primer, $2.5 \mathrm{U}$ of Taq DNA polymerase (Perkin Elmer AmpliTaq, PE Biosystems, Foster City, CA), $200 \mu \mathrm{m}$ of each deoxynucleotide triphosphate (dNTPs), $5 \mu \mathrm{l}$ of $10 \times$ reaction buffer, $4.5 \mathrm{mM} \mathrm{MgCl}_{2}$, and either $50 \mathrm{ng}$ of fungal DNA or 5 to $10 \mathrm{ng}$ of plasmid DNA as template. Amplifications were performed in a thermal cycler (Robocycler Gradient 40 Temperature Cycler, Stratagene, La Jolla, CA) programmed for an initial denaturation of $3 \mathrm{~min}$ at $95^{\circ} \mathrm{C}$, followed by 35 cycles of denaturation at $94^{\circ} \mathrm{C}$ for $45 \mathrm{~s}$, annealing at $59^{\circ} \mathrm{C}$ for $1 \mathrm{~min}$, and extension at $72^{\circ} \mathrm{C}$ for $2.5 \mathrm{~min}$. At the end of these cycles, an additional incubation for $7 \mathrm{~min}$ at $72^{\circ} \mathrm{C}$ ensured complete extension. PCR products were separated in $0.7 \%$ agarose dissolved in $0.5 \times$ TBE buffer ( $45 \mathrm{mM}$ Trisborate, $1 \mathrm{mM}$ EDTA, $\mathrm{pH}$ 8.0) by electrophoresis, stained with ethidium bromide, and photographed (Polaroid Corp., Cambridge, MA; film types 667 or 55) under UV light at $304 \mathrm{~nm}$ with a Wratten 22A filter. The amplified avenacinase-like gene fragments were excised, purified (Gel Extraction Kit, QIAGEN Inc., Valencia, CA), and cloned into plasmid pCR 2.1-TOPO (TOPO TA Cloning kit, Invitrogen Corporation, Carlsbad, CA). Recombinant plasmids consistent with the size expected for inserted avenacinase-like gene fragments were confirmed by EcoRI restriction endonuclease digestion, PCR, and sequence analyses. Two additional internal primers (5' seq and $3^{\prime}$ seq; see annealing sites in Figure 1) were designed and used for sequencing.

DNA sequencing. Clones containing plasmids with the PCR fragments inserted were purified (Qiaquick Gel Extraction

Table 1. Fungal isolates used in this study

\begin{tabular}{|c|c|c|c|}
\hline Isolate & Host & $\begin{array}{c}\text { Source } \\
\text { (location) }\end{array}$ & $\begin{array}{l}\text { Source (refer- } \\
\text { ence) }\end{array}$ \\
\hline \multicolumn{4}{|c|}{ Gaeumannomyces graminis var. tritici } \\
\hline CB1 & Triticum aestivum $\mathrm{L}$. & Bedford Co., VA, USA & (8) \\
\hline CD1 & T. aestivum & Dinwiddie Co., VA, USA & (8) \\
\hline CE1 & T. aestivum & Essex Co., VA, USA & (8) \\
\hline $\mathrm{CH} 1$ & T. aestivum & Hanover Co., VA, USA & (8) \\
\hline $\mathrm{CHe} 1$ & T. aestivum & Henrico Co., VA, USA & (8) \\
\hline CK1a & T. aestivum & King and Queen Co., VA, USA & (8) \\
\hline CK1b & T. aestivum & King and Queen Co., VA, USA & (8) \\
\hline CS1 & T. aestivum & Suffolk Co., VA, USA & (8) \\
\hline M1 & T. aestivum & Montana, USA & D. Mathre ${ }^{\mathrm{a}}$ \\
\hline ATCC 28230 & T. aestivum & United Kingdom & $\mathrm{ATCC}^{\mathrm{b}}$ \\
\hline 3053 & T. aestivum & Washington, USA & M. Elliott ${ }^{c}$ \\
\hline 3055 & T. aestivum & Oregon, USA & M. Elliott \\
\hline 3056 & T. aestivum & Indiana, USA & M. Elliott \\
\hline 3060 & T. aestivum & Idaho, USA & M. Elliott \\
\hline 3066 & T. aestivum & Montana, USA & M. Elliott \\
\hline \multicolumn{4}{|l|}{ G. graminis var. avenae } \\
\hline ATCC 15419 & Avena sativa $\mathrm{L}$. & United Kingdom & ATCC \\
\hline PG-W & Agrostis stolonifera $\mathrm{L}$. & Pinegrove, Canada & H.C. Wetzel III \\
\hline FR-W & A. stolonifera & France & H.C. Wetzel III \\
\hline $\mathrm{RB}-\mathrm{W}$ & A. stolonifera & Delaware, USA & H.C. Wetzel III \\
\hline RI-W & A. stolonifera & Rhode Island, USA & H.C. Wetzel III \\
\hline WW-W & A. stolonifera & Ohio, USA & H.C. Wetzel III \\
\hline \multicolumn{4}{|c|}{ G. graminis var. graminis } \\
\hline ATCC 12761 & T. aestivum & United Kingdom & ATCC \\
\hline FL-19 & $\begin{array}{l}\text { Cyanodon dactylon } \\
\text { (L.) Pers. }\end{array}$ & Florida, USA & M. Elliott \\
\hline FL-39 & $\begin{array}{l}\text { Stenotaphrum secund- } \\
\text { ataum (Walt.) Kuntze }\end{array}$ & Florida, USA & M. Elliott \\
\hline FL-175 & Oryza sativa $\mathrm{L}$. & Florida, USA & M. Elliott \\
\hline 2033 & Glycine $\max (\mathrm{L}$.$) Merr.$ & Florida, USA & M. Elliott \\
\hline \multicolumn{4}{|c|}{ Gaeumannomyces cylindrosporus } \\
\hline ATCC 64420 & Poa pratensis $\mathrm{L}$. & Rhode Island, USA & M. Elliott \\
\hline $\begin{array}{l}\text { Phialophora radicicola } \\
\text { ATCC } 64414\end{array}$ & P. pratensis & Rhode Island, USA & M. Elliott \\
\hline Phialophora sp. & Unknown & Unknown & M. Elliott \\
\hline $\begin{array}{l}\text { Cercospora zeae-maydis } \\
\text { FOO1 }\end{array}$ & Zea mays $\mathrm{L}$. & Wythe Co., VA, USA & E.L. Stromberg \\
\hline
\end{tabular}

${ }^{a}$ Don E. Mathre, Department of Plant Sciences, Montana State University-Bozeman, Bozeman 59717-3150.

b American Type Culture Collection, 10801 University Boulevard, Manassas 20110-2209.

${ }^{c}$ Monica L. Elliott, Fort Lauderdale Research and Education Center, University of Florida, Ft. Lauderdale 33314-7799.

${ }^{d}$ Henry C. Wetzel III, Department of Plant Pathology, Kansas State University, Manhattan 665065502 . 
Primer AV1>

$\nabla$

1 AGATGTTGCGCTCAAGTGCTTTCGCTCTCCTCGCCTGGGCCTCCCTCTCGGAAGCCCAGTTCGGCATCAAGCATACTCAG Gga U35463 AGATGTTGCGCTCAAGT'GCTTTCGCTCTCCTCGCCTGGGCCTCCCTCTCGGAAGCCCACTTCGGCATCAAGCATACTCAG Gga ATCC 15419 AGATGTTGCGCTCAAGTGCTTTCGCTCTCCTCGCTTGGGCCTCCCTCTCGGAAGCCCAGTTCGGCATCAAGCATACTCAG Ggg ATCC 12761 AGATGTTGCGCTCAAGTGCTTTCGCTCTCCTCGCTTGGGCCTCCCTCTCGGAAGCCCAGTTCGGCATCAAGCATACTCAG Ggt ATCC 28230 AGATGTTGCGCTCAAGTGCTTTCGCTCTCCTCGCTTGGGCCTCCCTCTCGGAAGCCCAGTTCGGCATCAAGCATACTCAG Ggt M1 AGATGTTGCGCTCAAGTGCTTTCGCTCTCCTCGCTTGGGCCTCCCTCTCGGAAGCCCAGTTCGGCATCAAGCATACTCAG Gqt CB1 AGATGTTGCGCTCAAGTGCTrTCGCTCTCCTCGCTTGGGCCTCCCTCTCGGAAGCCCAGTTCGGCATCAAGCATACTCAG Ggt CH1

81 TATGGCACGAGCGAGCCTGTCTACCCGTCGCgtacgttatcaacaagccgaaagccttccgcagaccatcccacttttt Gga U35463 TATGGCACGAGCGAGCCTGTCTACCCGTCGCgtacgttatcaacaagccoaaagccttccgcagaccatcccactttttt Gga ATCC 15419 TATGGCACGAGCGAGCCTGTCTACCCGTCGCgtacgttgtcaacaagccaaaagccttccgcagaccatcccactttttt Ggg ATCC 12761 TATGGCACGAGCGAGCCTATCTACCCGTCGCgtgcgttgtcaacaagccaaaagccttccgcagaccatcccactttttt Ggt ATCC 28230 TATGGCACGAGCGAGCCTGTCTACCCGTCGCgtacgttgtcaacaagccaaaagccttccgcagaccatcccactttttt Ggt M1 TATGGCACGAGCGAGCCTGTCTACCCGTCGCgtacgtagtcaacaagccaaaagccttccgcagaccatcccactttttt Ggt CB1 TATGGCACGAGCGAGCCTGTCTACCCGTCGCgtacgttgtcaacaagccaaaagccttccgcagaccatcccactttttt Ggt CH1

161 ----ctgtctcgtacttgtgctaatcttctcgcacctctaGCCGAATCTCTGGCTCTGGAGGATGGGAAGCTGGCCTGG Gga U35463 ----ctgtctcgtacttgtgctaatcttctcgcacctctaGCCGAAATCTCTGGCTCTGGAGGATGGGAAGCTGGCCTGG Gga ATCC 15419 tt--ctgtctcgtacttgtgctaatctgctcgcacctctaGCCGAAATCTTGGGCTCTGGAGGATGGGAAGCTGGCCTGG Ggg ATCC 12761 t--ctgtctcgtcettgtgctaatctgctcgcacctctaGCCGAAATCTCGGGCTCTGGAGGATGGGAAGCTGGCCTGG Ggt ATCC 28230 ttt-ctgtctcgtact tgtgctaatctgctcgcacctctaGCCGAAATCTCGGGCTCTGGAGGATGGGAAGCTGGCCTGG Ggt M1 ttt-ctgtctcgtacttgtgctaatctgctcgcacctctaGCCGAAATCTCGGGCTCTGGAGGATGGGAAGCGGGCCTGG Ggt CB1 ttttctgtctcgtacttgtgctaatctgctcgcacctctaGCCGAATCTCGGGCTCTGGAGGATGGGAAGCTGGCCTGA Ggt CH1

$\square$

Ggg-specific primer>

241 CCAAAGCCAAGGACTTCGTCGCGCAACTGACGCCCGAGGAGAAGGCGAACATGGTCACAGGCACCCCCGGTCCCTGCGTG Gga U35463 CCAAAGCCAAGGACTTCGTCGCGCAACTGACGCCCGAGGAGAAGGCGAACATGGTCACAGGCACCCCCGGTCCCTGCGTG Gga ATCC 15419 CCAAAGCCAAGGACTTCGTCGCGCAGCTGACGCCCGAGGAGAAGGCGAACATGGTCACGGGCACCCCCGGTCCCTGCGTA Ggg ATCC 12761 CCAAAGCCAAGGACTTCGTCGCGCAGCTGACGCCCGAGGAGAAGGCGAACATGGTCACGGGCACCCCCGGTCCCTGCGTA Ggt ATCC 28230 CCAAAGCCAAGGACTTCGTCGCGCAGCTGACGCCCGAGGAGAAGGCGAACATGGTCACGGGCACCCCCGGTCCCTGCGTG Ggt M1 CCAAAGCCAAGGACTTCGTCGCGCAGCTGACGCCCGAGGAGAAGGCGAACATGGTCACGGGCACCCCCGGTCCCTGCGTG Ggt CB1 CCAAAGCCAAGGACTTCGTCGCGCAGCTGACGCCCGAGGAGAAGGCGAACATGGTCACGGGCACCCCCGGTCCCTGCGTG Ggt CH1

$\square \nabla$ $\nabla \nabla \square \nabla$ $\nabla$

321 GGCAACATCGCCCCCGTGCCGCGCCTCAACTTCACCGGCCTGTGCCTACAGGACGGGCCGGCCACCCTCCGCCAGGCCAC Gga U35463 GGCAACATCGCCCCCGTGCCGCGCCTCAACTTCACCGGCCTGTGCCTACAGGACGGGCCGGCCACCCTCCGCCAGGCCAC Gga ATCC 15419 AGCAACATCGCCCCCGTGCCGCGCCTCAACTTCACCGGCCTGTGCCTACAGGACGGCCCGGCCACCCTCCGCCAGGCCAC Ggg ATCC 12761 GGCAACATCGCCCCCGTGCCGCGCCTCAACITCACCGGCCTGTGCCTACGGGACGGCCCGGCCACCCTCCGCCAGGCCAC Ggt ATCC 28230 GGCAACATCGCCCCCGTGCCGCGCCTCAACTTCACCGGGCTGTGCCTACAGGACGGCCCGGCCACCCTCCGCCAGGCCAC Ggt M1 GGCAACATCGCCCCCGTGCCGCGCCTCAACTTCACCGGGCTGTGCCTACAGGACGGCCCGGCCACCCTCCGCCAGGCCAC Ggt CB1 GGCAACATCGCCCCCGTGCCGCGCCTCAACTTCACCGGGCTGTGCCTACAGGACGGCCCGGCCACCCTCCGCCAGGCCAC Ggt CH1

401 TTACGTCACCGTCTTCCCGGGCGGTGTCAGCGCGGCTTCGTCGTGGGACAAGGACCTCATCTACAAGCACGGCGTGCTGA Gga U35463 TTACGTCACCGTCTTCCCGGGCGGTGTCAGCGCGGCTTCGTCGTGGGACAAGGACCTCATCTACAAGCACGGCGTGCTGA Gga ATCC 15419 TTACGTCACCGTCTTCCCGGGCGGTGTCAGCGCGGCTTCGTCGTGGGACAAGGACCTCATCTACAAGCACGGCGTGCTGA Ggg ATCC 12761 TTACGTCACCGTCTTCCCCGGCGGTGTCAGCGCGGCPTCGTCGTGGGACAAGGACTTCATTTACAAGCGCGGCGTGCTGA Ggt ATCC 28230 TTACGTCACCGTCTTCCCGGGCGGTGTCAGCGCGGCTTCGTCGTGGGACAAGGACCTCATCTACAAGCACGGCGTGCTGA Ggt M1 TTACGTCACCGTCTTCCCGGGCGGTGTCAGCGCGGCTTCGTCGTGGGACAAGGACCTCATCTACAAGCACGGCGTGCTGA Ggt CB1 TTACGTCACCGTCTTCCCGGGCGGTGTCAGCGCGGCTTCGTCGTGGGACAAGGACCTCATCTACAAGCACGGCGTGCTGA Ggt CHI

$\nabla$

Ggt-specific primer>

$\square$

481 TGGCCGAGGAGTTCCGTGACAAGGGGTCCCACGTCATCCTCGGCCCTGTAATTGGTCCCCGTGGAAGGTCCCCGTACGCC Gga U35463 TGGCCGAGGAGT TCCGTGACAAGGGGTCCCACGTCATCCTCGGCCCTGTAATTGGTCCCCGTGGAAGGTCCCCGTACGCC Gga ATCC 15419 TGGCCCGAGAGTTCCGTGACAAGGGCTCTCACATCATCCTCGGCCCTGTAATTGGTCCCCTTGGAAGGTCCCCGTACGCC Ggg ATCC 12761 TGGCCCGAGAGTTCCGTGACAAGGGCTCTCACATCATCCTCGGCCCTGTAATTGGTCCCCTTGGAAGGTCCCCGTACGCC Ggt ATCC 28230 TGGCCCGAGAGTTCCGTGACAAGGGCTCTCACATCATCCTCGGCCCCGTAATTGGCCCCCTTGGAAGGTCCCCGTACGCC Ggt M1 TGGCCCGAGAGTTCCGTGACAAGGGCTCTCACATCATCCTCGGCCCCGTAATTGGCCCCCTTGGAAGGTCCCCGTACGCC Ggt CB1 TGGCCCGAGAGTTCCGTGACAAGGGCTCTCACATCATCCTCGGCCCCGTAATTGGCCCCCTTGGAAGGTCCCCGTACGCC Ggt CH1

561 GGGCGCAACTGGGAGGGATTCTCCCCGACTTCGTACCTCGCGGGCGTCATGGCAGAGCAGACGGTCAAGGGGATGCAGGt Gga U35463 GGGCGCAACTGGGAGGGATTCTCCCCGACTTCGTACCTCGCGGGCGTCATGGCAGAGCAGACGGTCAAGGGGATGCAGGt Gga ATCC 15419 GGGCGCAACTGGGAGGGATTCTCCCCCGACTCGTACCTCGCGGGCGTCCTGGCAGAGCAGACGGTCAAGGGGATGCAGGt Ggg ATCC 12761 GGGCGCAACTGGGAGGGATTCTCCCCCGACTTGTACCTCGCGGGCGTCCTGGCAGAGCAGACGGTCAAGGGGATGCAGGt Ggt ATCC 28230 GGGCGCAACTGGGAGGGATTCTCCCCCGACTCGTACCTCGCGGGCGTCCTGGCAGAGCAGACGGTCAAGGGGATGCAGGt Ggt M1 GGGCGCAACTGGGAGGGATTCTCCCCCGACTCGTACCTCGCGGGCGTCCTGGCAGAGCAGACGGTCAAGGGGATGCAGGt Ggt CB1 GGGCGCAACTGGGAGGGATTCTCCCCCGACTCGTACCTCGCGGGCGTCCTGGCAGAGCAGACGGTCAAGGGGATGCAGGt Ggt CH1

(continued on next page)

Fig. 1. Aligned partial avenacinase and avenacinase-like nucleotide base sequences. The upstream $\left(5^{\prime}\right)$ sequence $(1$ to $1,385 \mathrm{bp})$ of the $3,143 \mathrm{bp}$ of the complete Gaeumannomyces graminis var. avenae ( $\mathrm{Gga}$ ) avenacinase gene (GenBank accession U35463 from Osborne et al. [28]) is compared with those for the avenacinase gene from Gga isolate ATCC 15419 (GenBank accession AF365953) and avenacinase-like genes from G. graminis var. graminis (Ggg; ATCC 12761 [AF365954]) and var. tritici (Ggt; ATCC 28230 [AF365956], M1 [AF365958], CB1 [AF365956], and CH1 [AF365957]). Nucleotides differing from the U35463 sequence are indicated in bold print. Nucleotides in intron regions are shown in lowercase. Sequences were generated by polymerase chain reaction (PCR) amplification using primers AV1 and AV3. Annealing sites for primers (AV1, AV2, AV3, Gga-specific, Ggg-specific, Ggtspecific, and internal primers $5^{\prime}$ and $3^{\prime}$ used for sequencing) are indicated by underlining. Consensus restriction endonuclease sites in all Gga, Ggg, and Ggt sequences are indicated with open symbols, nonconsensus sites are indicated with filled symbols: AluI $\left(\mathrm{O}, \boldsymbol{\bullet} ; 5^{\prime}\right.$-AGCT-3'), HaeIII ( $\nabla, \mathbf{\nabla} ; 5^{\prime}$-GGCC$\left.3^{\prime}\right)$, and $M s p \mathrm{I}\left(\square, \square ; 5^{\prime}-\mathrm{CCGG}-3^{\prime}\right)$. The single EcoRI restriction site is indicated. Alignments were performed using the CLUSTAL method with Weighted residue weight table. Hyphens were used to maximize the alignment. 
Fig. 1. (continued from preceding page)

641. aaggacccctctccaccaacatgtcggcgccgagcctattacccotaatactgacactt-gacagTCGGTCGGCGTGCA Gga U35463 aaggaccctctccaccaacatgtcggcgccgagcctattaccccgtaatactgacactt-gacagTCGGTCGGCGTGCA Gga ATCC 15419 aaggggccctctccagcaacatgttggcgccgagcctatt-ccctgtaatactgacactttgacagTCGGTCGGCGTGCA Ggg ATCC 12761 aaggggccctctccagcaacatgttggcgccgagcctatt-ccctgtaatactgacactttgacagTCGGTCGGCGTGCA Ggt ATCC 28230 aaggagccctctccagcaacatgtcggcgccgagcctatt-ccctgtaatactgacactttgacagTCGGTCGGCGTGCA Ggt M1 aaggagccctctccagcaacatgtcggegccgagcctatt-ccctgtaatactgacactttgacagTCGGTCGGCATGCA Ggt CBI aaggagccctctccagcaacatgtcggcgccgagcctatt-ccctgtaatactgacactttgacagTCGGTCGGCGTGCA Ggt CH1

$<$ Primer AV2

5' sequencing primer

Gga-specific primer>

$\nabla$

721 AGCCTGCACCAAGCACTTCATCGGCAATGAGCAGGAGGAGCAGCGCAACCCCACGGCGGTGGATGGCAAGACGGTTGAGG Gga U35463 AGCCTGCACCAAGCACTTCATCGGCAATGAGCAGGAGGAGCAGCGCAACCCCACGGCGGTGGATGGCAAGACGGTTGAGG Gga ATCC 15419 AACCTGCACCAAGCACTTCATCGGCAATGAGCAGGAGGAGCAGCGCAACCCCACGACGGTGGATGGCAAGGGGGTTGAGG Ggg ATCC 12761 AACCTGCACCAAGCACTTCATCGGCAATGAGCAGGAGGAGCAGCGCAACCCCACGACGGTGGATGGCAAGGGGGTTGAGG Ggt ATCC 28230 AACCTGCACCAAGCACTACATCGGCAATGAGCAGGAGGAGCAGCGCAACCCCACGACGGTGGATGGCAAGGGGGTTGAGG Ggt M1 AACCTGCACCAAGCACTACATCGGCAATGAGCAGGAGGAGCAGCGCAACCCCACGACGGTGGATGGCAAGGGGGTTGAGG Ggt CBI AACCTGCACCAAGCACTACATCGGCAATGAGCGGGAGGAGCAGCGCAACCCCACGACGGTGGATGGCAGGGGGGT'TGAGG Ggt CH1

801 CCATCTCGTCCAACATTGATGACCGCACAATGCACGAGGCCTACCTGTGGCCCTTTTACAACGCCGTCAGGGCCGGCACC Gga U35463 CCATCTCGTCCAACATTGATGACCGCACAATGCACGAGGCCTACCTGTGGCCCTTTTIACAACGCCGTCAGGGCCGGCACC Gga ATCC 15419 CCATCTCGTCCAACATTGACGACCGCACAATGCACGAGACCTACCTGTGGCCCTTTTACAACGCCGTCAGGGCCGGCACC Ggg ATCC 12761 CCATCTCGTCCAACATTGACGACCGCACAATGCACGAGACCTACCTGTGGCCCTTTTACAACGCCGTCAGGGCCGGCACC Ggt ATCC 28230 CCATCTCGTCCAACATTGACGACCGCACAATGCACGAGACCTACCT'GTGGCCCTTTTACAACGCCGTCAGGGCCGGCACC Ggt M1 CCATCTCGTCCAACATTGACGACCGCACAATGCACGAGACCTACCTGTGGCCCTTTTACAACGCCGTCAGGGCCGGCACC Ggt CBI CCATCTCGTCCAACATTGACGACCGCACAATGCACGAGACCTACCTGTGGCCCTr'T'TACAACGCCGTCAGGGCCGGCACC Ggt CH1

881 ACCTCCATAATGTGCTCTTACCAGAGGATCAACGGCAGCTACGGTTGTCAGAACAGCAAGACCCTCAACGGGCTTCT-CA Gga U35463 GCCTCCATAATGTGCTCTTACCAGAGGATCAACGGCAGCTACGGCTGCCAGAACAGCAAGACCCTCAACGGGCTTCT-CA Gga ATCC 15419 ACGTCCATAATGTGCTCTTACCAGAGGATCAACGGCAGCTACGGCTGCCAGAACAGCAAGACCCTCAACGGGCTTCT-CA Ggg ATCC 12761 ACCTCCATAATGTGCTCTTACCAGAGGATCAACGGCAGCTACGGCTGCCAGAACAGCAAGACCTTCAACGGGCTTCTTCA Ggt ATCC 28230 ACGTCCATAATGTGCTCTTACCAGAGGATCAACGGCAGCTACGGCTGCCAGAACAGCAAGACCCICAACGGGCTTCT-CA Ggt M1 ACGTCCATAATGTGCTCTTACCAGAGGATCAACGGCAGCTACGGCTGCCAGAACAGCAAGACCCTCAACGGGCTTTT-CA Ggt CB1 ACGTCCATAATGTGCTCTTACCAGAGGATCAACGGCAGCTACGGCTGCCAGAACAGCAAGACCCTCAACGGGCTTCT-CA Ggt CH1

O

$<3^{r}$ sequencing primer

961 AGACCGAGCTCGGCTTCCAGGGCTTCGTCGTGTCGGACTGgtgcgtggctacctccctctaccagatgaaacatgcagtg Gga U35463 AGACTGAGCTCGGCTTCCAGGGCTTCGTCGTGTCGGACTGgtgcgtggctacctccctctaccagatgaaacatgcagtg Gga ATCC 15419 AGACCGAGCTCGGCTTCCAGGGCTITCGTCGTGTCGGACTGgtgcgtggctacctccctctacCagatgaaacatgcagtg Ggg ATCC 12761 AGACCGAGCTCGGCTTCCAGGGCTTCGTCGTGTCGGACTGgtgcgtggctacctccttctaccagatgaaacatgcagtg Ggt ATCC 28230 AGACCGAGCTCGGCTTCCAGGGCTTCGTCGTGTCGGACTGgtgcgtggctacctecctctaccagatgaaacatgcagtg Ggt M1 AGACCGAGCTCGGCTTCCAGGGCTTCGTCGTGTCGGACTGgtgcgtggetacetccetctaccagatgaaacatgcagtg Ggt CB1 AGACCGAGCTCGGCTTCCAGGGCTTCGTCGTGTCGGACTGgtgcgtgget tcctccetctaccagatgaaacatgcagtg Ggt CH1 $\nabla$ $\square$

1041 cettgttttt-gctaatggccatgacagGGCCGCTACCCATTCCGGAGTTGCCTCCATTGAGGCTGGTCTGGACATGAAC Gga U35463 ccttgttttt-gctaatggtcataacagGGCCGCTACCCATTCCGGAGTTGCCTCCATTGAGGCTGGTCTGGACATGAAC Gga ATCC 15419 ccttgttttt-gctaatggccataacagGGCCGCTACCCATTCCGGAGTCGCCTCCATTGAGGCTGGTCTGGACATGAAC Ggg ATCC 12761 ccttgtttttgctaatggccataacagGGCCGCTACCCATTCCGGAGTCGCCTCCATTGAGGCTGGTCTGGACATGAAC Ggt ATCC 28230 ccttgttttt-gctaatggccataacagGGCCGCTACCCATTCCGGAGTCGCCTCCATTGAGGCTGGTCTGGACATGAAC Ggt M1 ccttgttttt-gctaatggccataacagGGCCGCTACCCATTCCGGAGTCGCCTCCATTGAGGCTGGTCTGGACATGAAC Ggt CB1 ccttgtttt-gctaatggccataacagGGCCGTACCCATTCCGGAGTCGCCTCCATTGAGGCTGGTCTGGACATGAAC Ggt CH1 $\square$

1121 ATGCCCGGACCGCTTAATTTTTTTGCCCAACCTTCGAGTCTrACTTTGGCAAGAACATCACCACTGCGGTCAACAACGG Gga U35463 ATGCCCGGATCGCTCGATTTTTTTGCCCCAACCTTCGAGTCTTACTTTGGCAAGAACATCACCACTGCGGTCAACAACGG Gga ATCC 15419 ATGCCCGGACCGCTCAATTTTTTTGCCCCAACCCTCGAGTCTTACTTTGGCAAGAACATCACCACTGCGGTCAACAACGG Ggg ATCC 12761 ATGCCCGGACCGCTCAATTTTTTTGCCCCAACCCTCGGGTCTTACTTTGGCAAGAACATCACCACTGCGGTCAACAACGG Ggt ATCC 28230 ATGCCCGGACCGCTCAATTTTTTTGCCCCAACCCTCGAGTCTTACTTTGGCAAGAACATCACCACTGCGGTCAATAACGG Ggt M1 ATGCCCGGACCGCTCAATTTTTTTGCCCCAACCCTCGAGTCTTACTTTGGCAAGAACATCACCACTGCGGTCAATAACGG Ggt CB1 ATGCCCGGACCGCTCAATTTTTTTGCCCCAACCCTCGAGTCTTACTTTGGCAAGAACATCACCACTGCGGTCAATAACGG Ggt CH1 $\square$

1201 CACACTCTCCTCCCGGAGGGTCGACGAGATGATTGAGCGCATCATGACTCCCTACTTCGCCCTGGGTCAGGACAAGAACT Gga U35463 CACACTCTCCTCCCGGAGGGTCGACGAGATGATTGAGCGCATCATGACTCCCTACTTCGCCCTGGGTCAGGACAAGAACT Gga ATCC 15419 CACACTCTCCTCCCGGAGGGTCGACGACATGATTGAGCGCATCATGACTCCCTACTTCGCCCTGGGTCAGGACAAGGACT Ggg ATCC 12761 CACACTCTCCTCCCGGAGGATCGACGACATGATTGAGCGCATCATGACTCCCTACTTCGCCCTGGGTCAGGACAAGGACT Ggt ATCC 28230 CACACTCTCCTCCCGGAGGGTCGACGACATGATTGAGCGCATCATGACTCCCTACTTCGCCCTGGGTCAGGACAAGGACT Ggt M1 CACACTCTCCTCCCGGAGGGTCGACGACATGATTGAGCGCATCATGACTCCCTACTTCGCCCTGGGTCAGGACAAGGACT Ggt CB1 CACACTCTCCCCCCGGAGGGCCGACGACATGATTGAGCGCATCATGACTCCCTACTTCGCCCTGGGTCAGGACAAGGACT Ggt CH 1

1281 ACCCCCCTGTCGACGGCTCCACGGTGTCCGTCGGCTTCTCGCAGCCCGGCTTCTGGAGCCACGAATTCCCCCTCGGCCCC Gga U35463 ACCCCCCTGTCGACGGCTCCACGGTGTCCGTCGGCTICTCGCGGCCCGGCTTCTGGAACCACGAATTCCCCCTCGGCCCC Gga ATCC 15419 ACCCCCCTGTCGACGGCTCCACGGTGCCCATCGGCTACTTGCAGCCCGACGCCTGGAACCACGAATTCCCCCTCGGCCCC Ggg ATCC 12761 ACCCCCCTGTCGACGGCTCCACGGTGCCCATCGGCTTCTTGCAGCCCGACGTCTGGAGCCACGAATTCCCCCTCGGCCCC Ggt ATCC 28230 ACCCCCCTGTCGACGGCTCCACGGTGCCCATCGGCTACTTGCAGCCCGACGCCTGGAACCACGAATTCCCCCTCGGCCCC Ggt M1 ACCCCCCTGTCGACGGCTCCACGGTGCCCATCGGCTACTTGCAGCCCGACGCCTGGAACCACGAATTCCCCCTCGGCCCC Ggt CB1 ACCCCCCTGTCGACGGCTCCACGGTGCCCATCAGCTACTTGCAGCCCGACGCCTGGAACCACGAATTCCCCCTCGGCCCC Ggt CH1

$<A V 3$ ( $3^{\prime}$ common primer)

1361 ACGGTCGACGTGCGCAGGAACCACCATGAGCA 1385 Gga U35463 ATGGTTGACGTGCGCAGGAACCACCATGAGCA 1385 Gga ATCC 15419 ACGGTCGACGTGCGCAGGAACCACCATGAGCA 1387 Ggg ATCC 12761 ACGGTCGACGTGCGCAGGAACCACCATGAGCA 1388 Ggt ATCC 28230 ACGGTCGACGTGCGCAGGAACCACCATGAGCA 1388 Ggt M1 ACGGTCGACGTGCGCAGGAACCACCATGAGCA 1389 Ggt CBI ACGGTCGACGTGCGCAGGAACCACCATGAGCA 1390 Ggt CH1 
Kit, QIAGEN, Inc., Valencia, CA) and sequenced using a dye-terminator cycle sequencing reaction based on the manufacturer's protocol (BigDye Terminator Cycle Sequencing, PE Applied Biosystem, Foster City, CA). Sequencing was performed with an ABI Prism DNA Sequencer model 373A Stretch (PE Applied Biosystem). Sequence data were analyzed with the aid of Lasergene Sequence Analysis Software (DNAStar Inc., Madison, WI) and on-line basic BLAST search (BLAST 2.0) provided by the National Center for Biotechnology Information.

PCR of Gga-, Ggg-, and Ggt-specific fragments. DNA was amplified using three $5^{\prime}$ primers, one specific for each vari- ety of G. graminis, and a single $3^{\prime}$ common primer. Annealing locations and nucleotide sequences for these primers on the avenacinase-like genes are shown in Figure 1. Primers used were synthesized by GIBCO BRL Custom Primers (Life Technologies Inc., Gaithersburg, MD). PCR reaction conditions differed from the procedures used to amplify avenacinase-like genes: a $50-\mu \mathrm{l}$ reaction mixture contained $25 \mathrm{pmol}$ of each of the three variety-specific primers, 75 pmol of $3^{\prime}$ common primer, $2.5 \mathrm{U}$ of Taq DNA polymerase (Qiagen Inc., Valencia, CA), $100 \mu \mathrm{M}$ of each dNTP, reaction buffer, $3 \mathrm{mM} \mathrm{MgCl}_{2}$, and $50 \mathrm{ng}$ of fungal DNA. PCR was performed in a thermal cycler (Mastercycler Gradient,

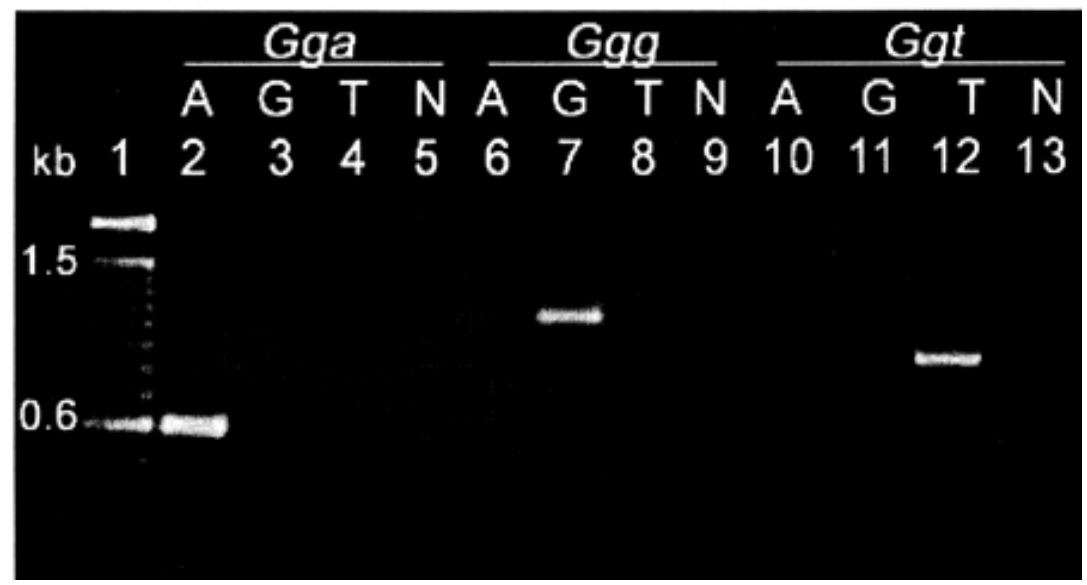

Fig. 2. Specificity of primers for templates. Variety-specific primers for Gaeumannomyces graminis var. avenae (Gga; lanes 2 to 5), var. graminis ( $\mathrm{Ggg}$; lanes 6 to 9), and var. tritici (Ggt; lanes 10 to 13) were tested against individual genomic DNA templates. Lanes 2, 6, and 10 contain Gga (ATCC 14519) template DNA (A); lanes 3, 7, and 11 contain Ggg (ATCC 12761) template DNA (G); lanes 4, 8, and 12 contain Ggt (M1) template DNA (T); and lanes 5, 9, and 13 contain no DNA (N). Lane 1 contains a 100-bp DNA ladder as a molecular weight standard. Ethidium bromide-stained varietyspecific polymerase chain reaction (PCR) products (Gga, $617 \mathrm{bp} ; G g g, 1,086 \mathrm{bp}$; and Ggt, $870 \mathrm{bp}$ ) were visualized by fluorescence with ultraviolet light.

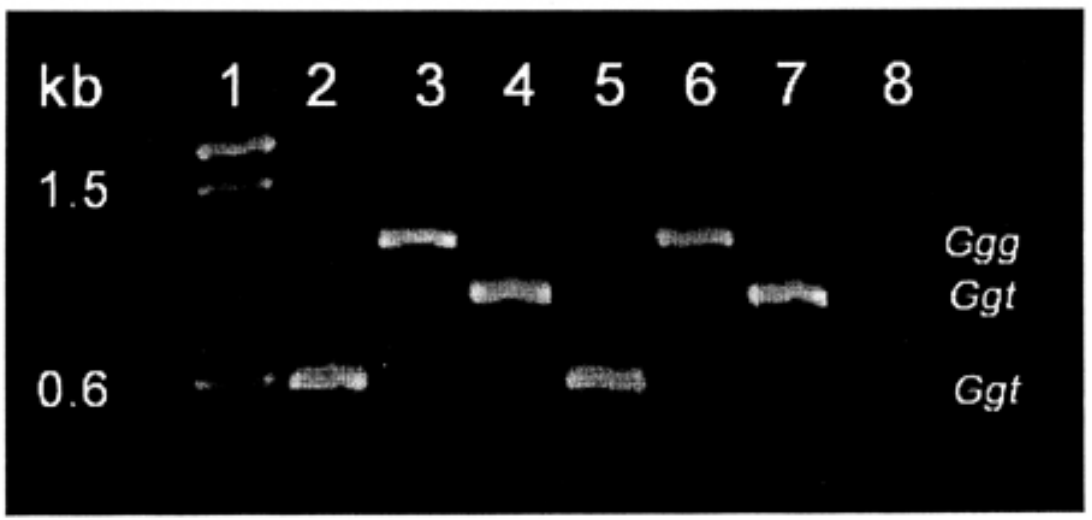

Fig. 3. Variety-specificity with mixed primers. Single template DNAs from Gaeumannomyces graminis var. tritici $(G g t)$, var. avenae $(G g a)$, and var. graminis $(G g g)$ primed with single and mixed variety-specific primers were used to amplify variety-specific polymerase chain reaction (PCR) products (Gga, $617 \mathrm{bp}$; $G g g, 1,086 \mathrm{bp}$; and $G g t, 870 \mathrm{bp}$ ). Lane 1 contains a 100-bp DNA ladder as a molecular weight standard. Lane 2 contains Gga (ATCC 14519) DNA amplified with Gga-specific primer. Lane 3 contains $G g g$ (ATCC 12761) DNA amplified with $G g g$-specific primer. Lane 4 contains Ggt (M1) DNA amplified with $G g t$-specific primer. Lanes 5 to 7 contain, respectively, Gga, $G g g$, and $G g t$ template DNAs. Lane 8 contains no DNA. Lanes 5 to 8 were amplified using a mixture of all three variety-specific primers. Ethidium bromide-stained PCR products were visualized by fluorescence with ultraviolet light.
Eppendorf Scientific Inc., Westbury, NY) programmed for an initial denaturation of 3 min at $95^{\circ} \mathrm{C}$, followed by 30 cycles, each consisting of denaturation at $94^{\circ} \mathrm{C}$ for $45 \mathrm{~s}$, primer annealing at $68^{\circ} \mathrm{C}$ for $45 \mathrm{~s}$, and extension at $72^{\circ} \mathrm{C}$ for $2 \mathrm{~min}$. At the completion of these cycles, an additional incubation for $10 \mathrm{~min}$ at $72^{\circ} \mathrm{C}$ ensured complete extension of PCR products. PCR products were separated by gel electrophoresis in $1.8 \%$ agarose. Negative controls with no DNA template were included in all PCR experiments. To control for falsepositives, some samples were amplified with only a single, variety-specific primer $(50 \mathrm{pmol})$.

Verification of primer specificity in PCR assays was carried out as described above with some modifications. If more than one template was added, the ratio of all templates was kept constant at $1: 1$ or $1: 1: 1$ with $50 \mathrm{ng}$ of each DNA. To demonstrate the sensitivity of a variety-specific primer, PCR was carried out with a dilution series of DNA template specific to each primer. The templates were diluted in sterile water, and the concentrations of DNA used ranged from $200 \mathrm{ng}$ to $1 \mathrm{pg}$. PCR cycling parameters were as described above in this section. To confirm that each amplified variety-specific fragment had the expected DNA sequence, PCR products were cloned and sequenced.

\section{RESULTS}

Cloning avenacinase-like genes. The presence of single copies of avenacinaselike genes was confirmed in $G g a$ and $G g t$ isolates by DNA:DNA hybridization (results not shown) using fungal genomic DNAs digested with EcoRI, HindIII, or $K p n I$ and probed with the 750-bp fragment, amplified using AV1 and AV2, from the authentic $G g a$ avenacinase gene cloned in plasmid pA3G2 $(3,28)$ using a kit (DIG Nucleic Acid Detection Kit, Roche Biochemicals, Indianapolis, IN). This plasmid was provided by Ann Osbourn, Sainsbury Laboratory, John Innes Centre for Plant Science Research, Norwich, UK.

Sequences of avenacinase-like genes. The published sequence for the Gga avenacinase gene (GenBank accession U35463) was compared with the sequences of the 1.4-kb fragments obtained from $\mathrm{Gga}$ isolate ATCC 15419 (GenBank accession no. AF365953), Ggg isolate ATCC 12761 (AF365954), and Ggt isolates M1 (AF365958), CB1 (AF365956), CH1 (AF365957), and ATCC 28230 (AF365955) (Fig. 1). These fragments varied from 1,387 to $1,390 \mathrm{bp}$. All sequences contained three introns, corresponding to the Gga sequence. Interestingly, the sequence for $G g t$ isolate ATCC 28230 most closely resembles the sequence for $\mathrm{Ggg}$ isolate ATCC 12761. Comparison of the deduced amino acid sequences showed that the avenacinase-like sequences of $G g t$ and $G g g$ were more closely 
related to each other ( $99.2 \%$ identity) than to $G g a$ ( $94.8 \%$ to $G g t$ and $94.6 \%$ to $G g g$ ) (data not shown). The avenacinase and avenacinase-like genes showed little homology with other genes in family 3 of glucosyl hydrolases such as $\beta$-glucosidase genes from Aspergillus kawachii (GenBank AB003470) and GTPase activation proteins of Cochliobolus heterostrophus (GenBank AF029913) (data not shown).

Design, specificity, and sensitivity of Ggt-, Gga-, and Ggg-specific primers. Three PCR primers were designed to be variety-specific from DNA sequences of the avenacinase-like genes of $G g t$ and $G g g$ and the avenacinase gene of Gga (Fig. 1). Variety specificity was established by designing these three primers, nearer the upstream end of the genes, with single or double base differences at the $3^{\prime}$ end of each primer. A primer common to all three sequences was used for the downstream end of the PCR product. With this strategy, PCR products amplified from Ggt-, Gga-, and Ggg-specific primers and the 3' common primer can be distinguished by size: 870,617 , and $1,086 \mathrm{bp}$, respectively.

Variety specificity of the $5^{\prime}$ primers was tested several ways. Using single templates and either one or all three specific $5^{\prime}$ primers, the expected single amplification products were observed (Figs. 2 and 3). When more than one specific primer was added into a PCR reaction, the amplification efficiency of the expected products was as high as for reactions with single specific primers (Fig. 3; compare intensity of ethidium bromide fluorescence in lanes 2 to 4 with lanes 5 to 7 ), suggesting that primers were not limiting. In addition, nonspecific product amplification was not observed in any of these experiments. However, less efficient amplification of longer PCR products, i.e., Ggg- and Ggtspecific fragments, was observed in reactions containing all three templates and all three variety-specific primers, possibly due to competition for dNTP substrates (data not shown). The experiments shown in Figures 2 and 3 were repeated with similar results.

Sensitivity was based on titration of template until amplification no longer produced ethidium bromide-stained variety-specific products visible as UV fluorescent bands in agarose gels (results not shown). The lowest concentration of DNA templates that generated products with $G g g$ - and $G g t$-specific primers was $100 \mathrm{pg}$. The $G g a$-specific primer amplified from 50 pg of template DNA.

PCR-based test for differentiating $\boldsymbol{G}$. graminis varieties. All isolates of $G$. graminis and related fungi tested in this study are listed in Table 1. When DNAs of 15 putative Ggt isolates were amplified with only the Ggt-specific primer, PCR products were obtained from CB1, CD1, CE1, CH1, CK1b, CS1, M1, 3053, and 3055. No Ggt-specific fragments were generated from isolates ATCC 28230, CHe1, CK1a, 3056, 3060, and 3066 (panel A of Figures 4 and 5). The atypical $G g t$ isolates that failed to generate $G g t$-specific fragments were subjected to diagnostic PCR with all three variety-specific primers in a single reaction; they all produced abundant Ggg-specific PCR fragments

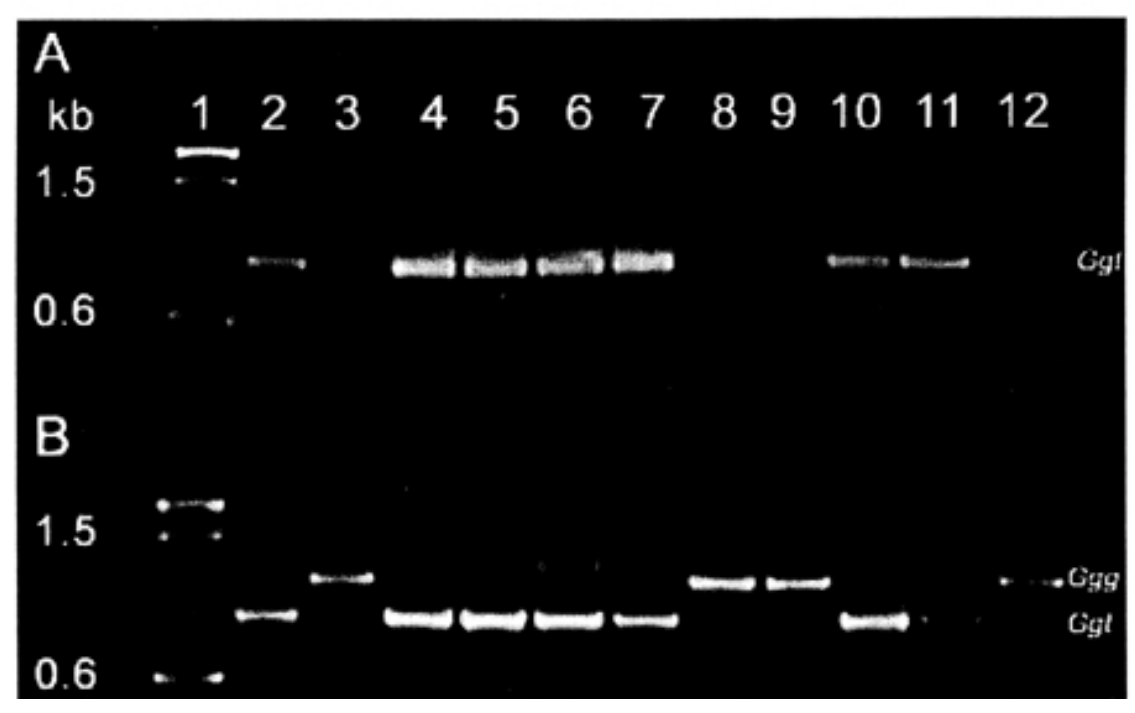

Fig. 4. Survey of Gaeumannomyces graminis var. tritici $(\mathrm{Ggt})$ isolates. Ggt isolates from wheat were amplified with $\mathbf{A}$, the $G g t$-variety specific primer, and $\mathbf{B}$, a mixture of all three variety-specific primers. Ethidium bromide-stained polymerase chain reaction (PCR) products (G. graminis vars. avenae [Gga], 617 bp; graminis [Ggg], 1,086 bp; and Ggt, $870 \mathrm{bp)}$ were visualized by fluorescence with ultraviolet light. Lane 1 contains a 100-bp DNA ladder as a molecular weight standard. For both panels, lanes 2 to 11 contain DNAs from Ggt isolates M1, ATCC 28230, CB1, CD1, CE1, CH1, $\mathrm{CHe} 1, \mathrm{CK} 1 \mathrm{a}, \mathrm{CK} 1 \mathrm{~b}$, and CS1, respectively. Lane 12, present only in B, contains DNA from $G g g$ ATCC 12761 as a control. The artifact above the major PCR product in $\mathbf{B}$, lane 6 , was not observed in two other amplifications with this DNA template.

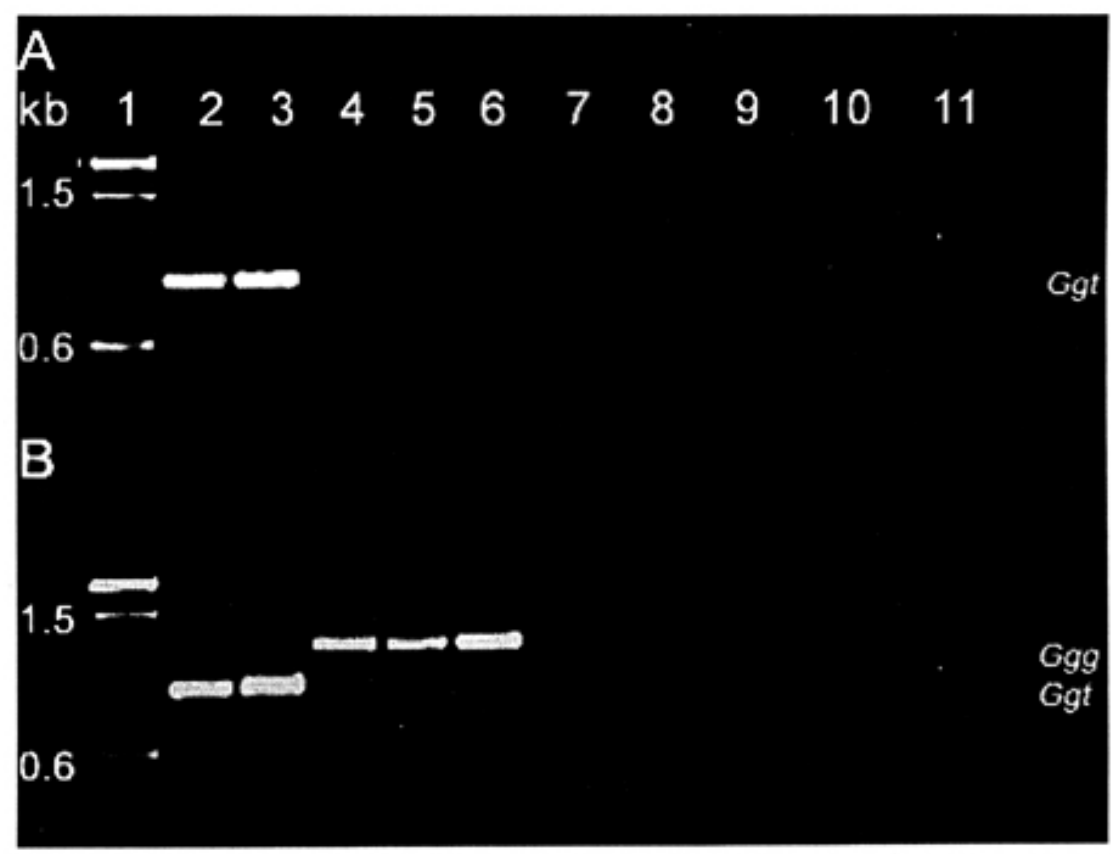

Fig. 5. Survey of isolates of the Gaeumannomyces-Phialophora complex. DNA from Gaeumannomyces graminis var. tritici $(G g t)$ isolates and other fungi were amplified using only the Ggt-specific primer (panel A) and all three variety-specific primers (G. graminis vars. avenae, Gga; graminis, Ggg; and Ggt) (panel B). Lane 1 contains a 100-bp DNA ladder as a molecular weight standard. For both panels, lanes 2 to 6 contain DNAs from $G g t$ isolates 3053, 3055, 3056, 3060, and 3066, respectively. Lanes 7 to 9 contain DNA from, respectively, Gaeumannomyces cylindrosporus ATCC 64420, Phialophora radicicola ATCC 64414, and Phialophora sp. Lane 10 contains DNA from Cercospora zeae-maydis F001, a fungus not related to the Gaeumannomyces-Phialophora complex. Lane 11 contains no DNA. Ethidium bromide-stained, variety-specific PCR products for $G g g, 1,086 \mathrm{bp}$, and Ggt, 870 bp, were visualized by fluorescence with ultraviolet light. 
(panel B of Figures 4 and 5). These six isolates also produced $\mathrm{Gg}$-specific fragments when amplified with only Gggspecific primer (data not shown). The experiments shown in Figures 4 and 5 were repeated with similar results.

To further characterize these atypical Ggt isolates, we studied restriction endonuclease digestion profiles of PCR products from the avenacinase-like genes. The predicted restriction endonuclease digestion sites of the partial avenacinaselike gene $(1.4 \mathrm{~kb})$ from one atypical $G g t$ isolate ATCC 28230 were identical to $G g g$ isolate ATCC 12761 (Fig. 1). Partial avenacinase-like gene PCR products $(1.4 \mathrm{~kb})$ from three of six atypical Ggt isolates, ATCC 28230, CK1a, and CHe1, were digested with the restriction endonucleases AluI, MspI, and HaeIII and separated and visualized as described above (see Figure 1

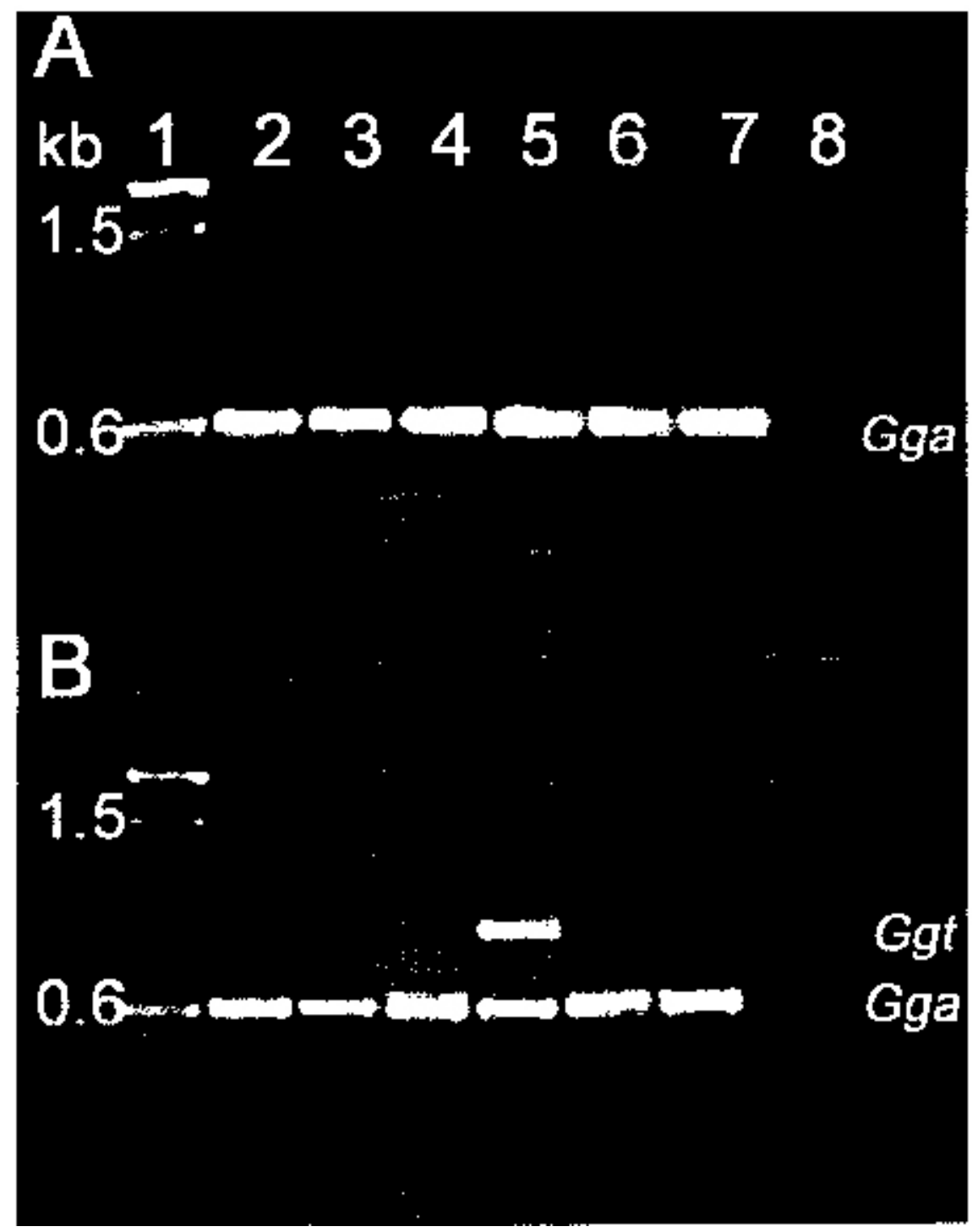

Fig. 6. Survey of Gaeumannomyces graminis var. avenae ( $G g a)$ isolates. Gga isolates with A, only the $G g a$-specific primer, and $\mathbf{B}$, all three variety-specific primers. Ethidium bromide-stained varietyspecific polymerase chain reaction (PCR) products $(G g a, 617$ bp and G. graminis var. tritici $[G g t]$, $870 \mathrm{bp}$ ) were visualized by fluorescence with ultraviolet light. Lane 1 contains 100-bp DNA ladder as a molecular weight standard. For both panels, lanes 2 to 8 contain DNA from Gga isolates ATCC 15419, FR-W, PG-W, RB-W, RI-W, WW-W, and no DNA, respectively. sample, one isolate, RB-W, produced two fragments of 870 and $617 \mathrm{bp}$, specific for Ggt and Gga, respectively (Fig. 6, panel B). This experiment was repeated with similar results for isolate RB-W. Ggg isolates from other plant species (Table 1) all amplified the 1,086-bp band but less efficiently than ATCC 12761 isolated from wheat. Ggg isolate, FL-19, which was isolated from bermudagrass (Cyanodon dactylon L.), generated a PCR product of $300 \mathrm{bp}$ that was not related by nucleotide sequence to any of the variety-specific fragments (data not shown). More Ggg isolates from wheat and other host plants should be tested before the significance of nontypical bands can be addressed. DNA sequences of $G g t$-, $G g a$-, and $G g g$-specific PCR fragments demonstrated that both $G g t$ - and Ggg-specific fragments were $100 \%$ identical to their avenacinase-like genes. However, the $\mathrm{Gga}$-specific fragment showed slight divergence (10 bp) from the published sequence of the avenacinase gene. This is not unexpected since Gga isolate ATCC 15419 used in this study was not the same isolate sequenced by Osbourn et al. (30).

\section{DISCUSSION}

We have identified large fragments (1.4 $\mathrm{kb})$ of genes for $G g t$ and $G g g$ that are very similar to $G g a$ avenacinase with less than 50 nucleotide base differences (95\% identity). This is the first report of partial DNA sequences for avenacinase-like genes in Ggt and Ggg isolates, although Osbourn et al. (30) briefly described the presence of avenacinase-like proteins produced by $G g t$ and Ggg. In addition, BLAST search results strongly indicated that avenacinaselike gene fragments closely resembled the Gga avenacinase gene in GenBank. We also show that the avenacinase-like genes of $G g t$ and $G g g$ are present as single copies as Bowyer et al. (3) found for avenacinase in Gga. Based on DNA sequence alignment products, Ggt isolates are more closely related to $G g g$ ( $99.2 \%$ identity) than Gga (94.6\%). We speculate that the ancestor of Gga, Ggg, and Ggt might have had an avenacinase-like gene. In the coevolution of $G g t$ and $G g g$ with their hosts, there was presumably no selective pressure on avenacinase-like genes since their hosts lacked avenacin. However, in the coevolution of $G g a$ with oats, the fungus evolved avenacinase from an avenacinase-like gene template for successful pathogenesis and survival. Avenacinase-like proteins may simply be $\beta$-glucosidases required for fungal nutrition, or they may play a role in detoxification of saponins during pathogenesis in other plants (3). Our avenacinase-like genes showed little similarity to $\beta$-glucosidase genes (data not shown). Isolation of the full-length genes and generation of mutants of Ggt and Ggg may be necessary in order to confirm the identity or to study the function of these genes. 
PCR technology offers a number of advantages over conventional methods for differentiation of take-all-causing varieties of G. graminis. Several groups have developed PCR-based methods for differentiation of varieties of G. graminis; however, most were based on amplification of the conserved regions of the ITS or 18S rDNA with either specific or random primers (4,14-16,37). In contrast, we demonstrate a PCR differentiation method based on a single gene target that is simple and specific for three varieties of take-all pathogens: G. graminis vars. avenae, graminis, and tritici.

Specificity of the PCR identification of $G g t, G g a$, and $G g g$ is based on nucleotide sequence differences among the genes encoding avenacinase or avenacinase-like proteins. Three upstream primers, annealing in the $5^{\prime}$ half of the genes, provide variety specificity by incorporating one to two mismatched bases at their $3^{\prime}$ end which do not anneal to the sequences of the nontarget varieties. A single common downstream primer, closer to the $3^{\prime}$ end of the genes, was paired with each of the variety-specific primers. Bryan et al. (4) successfully used similar primer design to amplify $G g a$ - and $G g t$-specific products from the ITS regions of rDNA.

An important feature of our differential identification test is that all three varietyspecific primers may be used in a single PCR reaction. In samples that amplify multiple bands, retesting with individual primer sets will eliminate the possibility of false-positive results. It should be noted that, based on PCR products from avenacinase-like genes, some fungi isolated from wheat tissues showing characteristic symptoms of take-all may be classified as Ggg, a common saprophyte or a weak pathogen on wheat. It is difficult to differentiate between Ggt and Ggg based on cultural appearance, especially since both of them grow on selective medium for Ggt. Use of ascospore size, perithecial characters, and hyphopodia shape to differentiate among these varieties is difficult because overlaps occur among all these traits $(20,35,38)$. Although mycological characters (ascospore, perithecia, and hyphopodia) were not part of this work, in a separate project in our laboratories, several of the strains used in this study were compared directly (8). Perithecial, ascospore, and hyphopodial type (nonlobed in all cases) differences among putative wheat $G g t$ isolates, ATTC 28230, CB1, CD1, CK1a, CHe1, and M1, were not great enough to suggest identification as Ggg rather than Ggt. However, in pathogenicity studies, Ggt isolates CD1, $\mathrm{CB} 1$, and $\mathrm{M} 1$, producing 870-bp PCR fragments, caused moderate to severe damage on wheat while Ggt isolates, ATCC 28230, CK1a, and $\mathrm{CHe} 1$, producing 1,086bp PCR fragments, caused mild damage (8). Our results confirm what others have stated or implied; it is difficult to differen- tiate between Ggt and Ggg (20,33-35,38). Although our survey, including 16 putative $G g t$ isolates and five putative $G g g$ isolates, is small for taxonomic purposes, it does suggest that identification difficulties may be a component in the variation of virulence among Ggt isolates.

Other closely related fungi such as $G$. cylindrosporus, Phialophora sp., and $P$. radicicola failed to amplify with any of the three variety-specific primers demonstrating primer specificity. This is one of the most important criteria for a test since these fungi are present together in the soil as a Gaeumannomyces-Phialophora complex $(9,10)$. The Gaeumannomyces-Phialophora complex also includes other morphologically similar fungi that are nonpathogenic on cereal roots $(34,35)$. $P$. radicicola produces lobed hyphopodia, while Phialophora spp. may produce either lobed or simple hyphopodia which cannot be distinguished from those of Ggg. Further confirmation with more isolates of Ggt, Gga, Ggg, Phialophora spp., and other closely related fungi such as $G$. incrustans and Magnaporthe spp. should be conducted to verify primer specificity.

The Gga- and Ggt-specific PCR products recovered from diverse isolates indicated a great deal of uniformity among $G g a$ populations, except for $G g a$ isolate RB-W. Using all three variety-specific primers, Ggt- and $G g a$-specific products were observed from isolate RB-W. When individual variety-specific primers were used with this isolate, expected PCR products were recovered with both $G g a$ - and $G g t$-specific primers. Two possible explanations exist. First, isolate RB-W may not be a pure culture, although it was cultured from a hyphal tip. Second, isolate RB-W may be a heterokaryon produced by anastomosis among G. graminis varieties. Further purification of the culture and sequencing of the products may determine the correct hypothesis.

The detection level for our diagnostic test was $100 \mathrm{pg}$ for $G g t$ and $G g g$, and 50 pg for Gga. In mixed DNA populations, PCR detection sensitivity decreased to $1 \mathrm{ng}$ of total DNA of each variety (data not shown). These levels are consistent with sensitivity for direct detection of the fungi from diseased plant tissues and soil samples (19). Those authors were able to detect $G g t$ directly from diseased wheat roots and infested soil samples using slot-blot hybridization with a mitochondrial DNA probe. They were able to detect DNA levels as low as 100 and $30 \mathrm{pg}$ in root and soil samples, respectively. In contrast, $\mathrm{Hu}$ et al. (22) showed that, with autoradiography, DNA of Verticillium albo-atrum (Rienke \& Berthold) from inoculated alfalfa (Medicago sativa L.) could be detected by amplification of the ITS regions down to $10 \mathrm{pg}$.

\section{ACKNOWLEDGMENTS}

We thank Joan Henson, Department of Microbiology, Montana State University, Bozeman 59717, and M. A. Saghai-Maroof, Department of
Crop and Soil Environmental Sciences (CSES), VPI\&SU, for their valuable discussions and advice on this work. We thank Ann Osbourn, Sainsbury Laboratory, John Innes Centre for Plant Science Research, Norwich, UK, for providing plasmid. We thank Alec Hayes, CSES, VPI\&SU, for his help in DNA sequencing. We also thank Monica L. Elliott, Fort Lauderdale Research and Education Center, University of Florida, Ft. Lauderdale 33314-7799; Henry C. Wetzel, Department of Plant Pathology, Kansas State University, Manhattan 66506-5502; and Don E. Mathre, Department of Plant Sciences, Montana State UniversityBozeman, Bozeman 59717-3150, for providing fungal isolates. This research was sponsored in part by the Virginia Small Grains Board; USDA, ARS Biocontrol of Plant Disease Laboratory, Beltsville, MD; and the Thai Government (scholarship to SR).

\section{LITERATURE CITED}

1. Asher, M. J. C. 1980. Variation in pathogenicity and cultural characters in Gaeumannomyces graminis var. tritici. Trans. Br. Mycol. Soc. 75:213-220.

2. Bateman, G. L., Ward, E., and Antoniw, J. F. 1992. Identification of Gaeumannomyces graminis var. tritici and G. graminis var. avenae using a DNA probe and non-molecular methods. Mycol. Res. 96:737-742.

3. Bowyer, P., Clarke, B. R., Lunness, P., Daniels, M. J., and Osbourn, A. E. 1995. Host range of plant pathogenic fungus determined by a saponin detoxifying enzyme. Science 267:371-374.

4. Bryan, G. T., Daniels, M. J., and Osbourn, A. E. 1995. Comparison of fungi within the Gaeumannomyces-Phialophora complex by analysis of ribosomal DNA sequences. Appl. Environ. Microbiol. 61:681-689.

5. Bryan, G. T., Labourdette, E., Melton, R. E., Nicholson, P., Daniels, M. J., and Osbourn, A. E. 1999. DNA polymorphism and host range in the take-all fungus, Gaeumannomyces graminis. Mycol. Res. 103:319-327.

6. Clarkson, J. D. S., and Polley, R. W. 1981. Diagnosis, assessment, crop-loss appraisal and forecasting. Pages 251-269 in: Biology and Control of Take-all. M. J. C. Asher and P. J. Shipton, eds. Academic Press, New York.

7. Crombie, W. M. L., Crombie, L., Green, J. B. and Lucas, J. A. 1986. Pathogenicity of 'takeall' fungus to oats: Its relationship to the concentration and detoxification of the four avenacins. Phytochemistry 25:2075-2083.

8. Crozier, J. B. 1999. Evaluation of agents for the suppression of take-all of wheat in Virginia in greenhouse and field studies. Ph.D. diss. Virginia Polytechnic Institute and State University, Blacksburg.

9. Deacon, J. W. 1974. Further studies on Phialophora radicicola and Gaeumannomyces graminis on roots and stem bases of grasses and cereals. Trans. Br. Mycol. Soc. 63:307327.

10. Deacon, J. W. 1981. Ecological relationships with other fungi: Competitors and hyperparasites. Pages 75-102 in: Biology and Control of Take-all. M. J. C. Asher and P. J. Shipton, eds. Academic Press, New York.

11. Elliott, M. L., Hagan, A. K., and Mullen, J. M. 1993. Association of Gaeumannomyces graminis var. graminis with a St. Augustinegrass root rot disease. Plant Dis. 77:206-209.

12. Elliott, M. L., and Landschoot, P. J. 1991. Fungi similar to Gaeumannomyces associated with root rot of turfgrasses in Florida. Plant Dis. 75:238-241

13. Epstein, L., Kaur, S., Goins, T., Kwon, Y. H., and Henson, J. M. 1994. Production of hyphopodia by wild-type and three transformants of Gaeumannomyces graminis var graminis. Mycologia 86:72-81.

14. Fouly, H. M., and Wilkinson, H. T. 2000. 
Detection of Gaeumannomyces graminis varieties using polymerase chain reaction with variety-specific primers. Plant Dis. 84:947951.

15. Fouly, H. M., Wilkinson, H. T., and Domier, L. L. 1996. Use of random amplified polymophic DNA (RAPD) for identification of Gaeumannomyces species. Soil Biol. Biochem. 28:703-710.

16. Goodwin, P. H., Hsiang, T., Xue, B. G., and Liu, H. W. 1995. Differentiation of Gaeumannomyces graminis from other turf-grass fungi by amplification with primers from ribosomal internal transcribed spacers. Plant Pathol. 44:384-391.

17. Henson, J. M. 1989. DNA probe for identification of the take-all fungus, Gaeumannomyces graminis. Appl. Environ. Microbiol. 55:284-288.

18. Henson, J. M. 1992. DNA hybridization and polymerase chain reaction (PCR) tests for identification of Gaeumannomyces graminis, Phialophora and Magnaporthe isolates. Mycol. Res. 96:269-273.

19. Herdina, P. H., and Ophel-Keller, K. 1996. Quantification of Gaeumannomyces graminis var. tritici in infected roots and soil using slot-blot hybridization. Mycol. Res. 100:962970

20. Hornby, D., Bateman, G. L., Gutteridge, R. J., Lucas, P., Osbourn, A. E., Ward, E., and Yarham, D. J. 1998. Take-All Disease of Cereals: A Regional Perspective. CAB International.

21. Hornby, D., Slope, D. B., Gutteridge, R. J., and Sivanesan, A. 1977. Gaeumannomyces cylindrosporus, a new ascomycete from cereal roots. Trans. Br. Mycol. Soc. 69:21-25.

22. Hu, X., Nazar, R. N., and Robb, J. 1993. Quantification of Verticillium biomass in wilt disease development. Physiol. Mol. Plant.
Pathol. 42:23-36.

23. Juhnke, M. E., Mathre, D. E., and Sands, D. C. 1984. A selective medium for Gaeumannomyces graminis var. tritici. Plant Dis. 68:233-236.

24. Landschoot, P. J., and Jackson, N. 1989. Gaeumannomyces incrustans sp. nov., a rootinfecting hyphopodiate fungus from grass roots in the United States. Mycol. Res. 93:5558.

25. Lee, S. B., and Taylor, J. W. 1990. Isolation of DNA from fungal mycelia and single spores. Pages 282-287 in: PCR Protocols: A Guide to Methods and Applications. M. A. Innis, D. H. Gelfand, J. J. Sninsky, and T. J. White, eds. Academic Press, San Diego.

26. Nilsson, H. E. 1972. The occurrence of lobed hyphopodia on an isolate of the take-all fungus, "Ophiobolus graminis Sacc.", on wheat in Sweden. Swedish J. Agric. Res. 2:105-118.

27. O'Dell, M., Flavell, R. B., and Hollins, T. W. 1992. The classification of isolates of Gaeumannomyces graminis from wheat, rye and oats using restriction fragment length polymophisms in families of repeated DNA sequences. Plant Pathol. 41:554-562.

28. Osbourn, A. E., Bowyer, P., Lunness, P., Clarke, B., and Daniels, M. 1995. Fungal pathogens of oat roots and tomato leaves employ closely related enzymes to detoxify different host plant saponins. Mol. PlantMicrobe Interact. 8:971-978.

29. Osbourn, A. E., Clarke, B. R., Dow, J. M., and Daniels, M. J. 1991. Partial characterization of avenacinase from Gaeumannomyces graminis var. avenae. Physiol. Mol. Plant Pathol. 38:301-312.

30. Osbourn, A. E., Clarke, B. R., Lunness, P., Scott, P. R., and Daniels, M. J. 1994. An oat species lacking avenacin is susceptible to infec- tion by Gaeumannomyces graminis var. tritici. Physiol. Mol. Plant Pathol. 45:457-467.

31. Schesser, K., Luder, A., and Henson, J. M. 1991. Use of polymerase chain reaction to detect the take-all fungus, Gaeumannomyces graminis, in infected wheat plants. Appl. Environ. Microbiol. 57:553-556.

32. Tan, M. K., Wong, P. T. W., and Holley, M. P. 1994. Characterization of nuclear ribosoma DNA (rDNA) in Gaeumannomyces graminis and correlation of rDNA variation with $G$. graminis varieties. Mycol. Res. 98:553-361.

33. Walker, J. 1972. Type studies on Gaeumannomyces graminis and related fungi. Trans. Br. Mycol. Soc. 58:427-457.

34. Walker, J. 1981. Taxonomy of take-all fungi and related genera and species. Pages 15-74 in: Biology and Control of Take-all. M. J. C. Asher and P. J. Shipton, eds. Academic Press, New York.

35. Ward, E., and Akrofi, A. Y. 1994. Identification of fungi in the Gaeumannomyces Phialophora complex by RFLPs of PCRamplified ribosomal DNAs. Mycol. Res. 98:219-224.

36. Ward, E., and Gray, R. M. 1992. Generation of a ribosomal DNA probe by PCR and its use in identification of fungi with the Gaeuman nomyces-Phialophora complex. Plant Pathol. 41:730-736.

37. Wetzel, H. C. III, Dernoeden, P H and Millner, P. D. 1996. Identification of darkly pigmented fungi associated with turfgrass roots by mycelial characteristics and RAPDPCR. Plant Dis. 80:359-364.

38. Yeates, J. S., Fang, C. S., and Parker, C. A 1986. Distribution and importance of oatattacking isolates of Gaeumannomyces graminis var. tritici in Western Australia. Trans. Br. Mycol. Soc. 86:145-152. 